\title{
Plasma B-cell activating factor levels and polymorphisms in hepatitis B-related hepatocellular carcinoma: Clinical correlation and prognosis
}

\author{
Apichaya Khlaiphuengsin, ${ }^{1}$ Natthaya Chuaypen, ${ }^{1}$ Nutcha Pinjaroen, ${ }^{2}$ Boonchoo Sirichindakul, ${ }^{3}$ \\ Nattiya Hirankarn, ${ }^{4}$ Pisit Tangkijvanich ${ }^{1}$
}

\begin{abstract}
Background: B-cell activating factor (BAFF), an important cytokine for B lymphocyte activation, has been shown to be increased in chronic hepatitis B virus (HBV) infection.
\end{abstract}

Objectives: This study aimed at evaluating clinical correlation and prognostic role of plasma BAFF and related polymorphisms in patients with HBV-related hepatocellular carcinoma (HCC).

Methods: Plasma BAFF levels were measured from 100 healthy controls and 490 patients with chronic HBV infection (200 with HCC and 290 without HCC). The rs9514828 and rs12583006 polymorphisms were determined by allelic discrimination.

Results: The HCC group had significantly higher BAFF levels compared with the non-HCC group and healthy controls. Among the non-HCC group, the HBeAg-positive subgroup had higher BAFF levels compared with the HBeAg-negative subgroup. In the HCC group, high BAFF levels at initial presentation significantly correlated with alpha-fetoprotein levels, Child-Pugh classification, tumor size and BCLC stage. Multivariate analyses showed that elevated BAFF concentration $(\geq 1,100 \mathrm{pg} / \mathrm{ml})$ was a significant and independent prognostic factor of overall survival in patients with HCC (OR $=2.28,95 \%$ CI: $1.07-4.87 ; P=0.034)$. HCC patients with high BAFF levels $(\geq 1,100 \mathrm{pg} / \mathrm{ml})$ had a poorer median survival than those with low levels $(P<0.001$, log-rank test). Regarding BAFF polymorphisms, the frequency of rs 9514828 $\mathrm{CT}+\mathrm{TT}$ genotypes was higher distributed in patients with chronic HBV infection compared with healthy controls (58.0\% vs. $46.0 \%, P=0.029)$.

Conclusions: Our data demonstrate for the first time that elevated plasma BAFF levels at baseline exhibit clinical correlation in terms of disease severity and overall survival in HCC patients. Thus, plasma BAFF at initial diagnosis could serve as a prognostic marker for HBV-related HCC.

Key words: BAFF, B cells, HBV, HCC, polymorphisms

\section{From:}

${ }^{1}$ Center of Excellence in Hepatitis and Liver Cancer,

Faculty of Medicine, Chulalongkorn University, Thailand

${ }^{2}$ Department of Radiology, Faculty of Medicine, Chulalongkorn

University, Thailand

Department of Surgery, Faculty of Medicine, Chulalongkorn University, Thailand

${ }^{4}$ Center of Excellence in Immunology and Immune-mediated Diseases, Faculty of Medicine, Chulalongkorn University, Thailand

\section{Corresponding author:}

Pisit Tangkijvanich

Center of Excellence in Hepatitis and Liver Cancer, Department of

Biochemistry, Faculty of medicine, Chulalongkorn University,

Bangkok, Thailand, 10330

Email: pisittkvn@yahoo.com

\section{Introduction}

Hepatitis B virus (HBV) infection is an essential public health problem worldwide. Individuals chronically infected with $\mathrm{HBV}$ have a diverse clinical manifestation including chronic hepatitis, progressive fibrosis, cirrhosis, and finally hepatocellular carcinoma (HCC) development. ${ }^{1}$ Accumulative recent evidence has suggested that the pathogenesis and consequence of HBV infection is greatly linked to immune-mediated host-virus interactions. ${ }^{2,3}$ During acute infection, vigorous and specific $\mathrm{B}$ and T-cell activities are coordinately involved in viral clearance and self-limited hepatitis. By contrast, the immune responses display functional impairment in individuals developing chronic $\mathrm{HBV}$ infection. ${ }^{2}$ 
Currently, it is documented that injury and eventually leads to the diversity of clinical outcome of HBV infection. ${ }^{4}$ Unlike the importance of $\mathrm{T}$-cell mediated immune response, data regarding the role of B-cell immunity in the pathogenesis and prognosis of chronic HBV infection are less well documented.

$\mathrm{B}$ cells exhibit an essential role in humoral immunity by producing antibodies, modulating inflammatory cytokines and inducing the T-cell response. ${ }^{5,6}$ In general, peripheral $\mathrm{B}$ lymphocytes require $B$ cell-activating factor (BAFF) for their activation, differentiation and survival. BAFF is a member of the tumor necrosis factor superfamily (TNFSF13B) and an IFN stimulated gene, which is produced by several cells such as neutrophils, monocytes, macrophages, dendritic cells and activated T cells. ${ }^{7}$ Previous reports have demonstrated that circulating BAFF levels are highly expressed and correlated with clinical activity and outcome of several autoimmune diseases and hematological malignancies. ${ }^{8-10}$ Apart from its established role in these diseases, enhanced BAFF levels were also observed in the context of many infections and non-hematological malignancies, suggesting it may play a pathogenic role in diverse disorders ${ }^{11}$ For instance, enhanced BAFF expression was detected in hepatitis $\mathrm{C}$ virus (HCV) infection, particularly among patients with mixed cryoglobulinemia. ${ }^{12,13}$ Moreover, it was recently shown that increased BAFF levels appeared to correlate with disease severity in patients with chronic HBV infection and was independently associated with the occurrence of HCC. ${ }^{14}$ These data suggest that BAFF may contribute to progressive liver disease and HCC development in patients with chronic HBV infection. However, its correlation with clinical characteristics and prognosis of HCC has not yet been completely evaluated.

Regarding host genetic variation, single nucleotide polymorphisms (SNPs) of the BAFF gene, including rs9514828 and rs12583006, have been shown to be associated with alteration of BAFF expression and linked to several autoimmune and hematological disorders. ${ }^{15-17}$ As available data in chronic HBV infection are limited, ${ }^{18}$ it is unclear whether these SNPs might be associated with clinical severity and prognosis of patients with HCC. To address these issues, we determined whether plasma BAFF and these polymorphisms were associated with clinical characteristics and outcome in patients with HBV-related HCC.

\section{Methods \\ Patients}

Stored samples for the measurement of plasma BAFF levels and polymorphisms were obtained from patients who were diagnosed of HBV-related HCC for the first time at King Chulalongkorn Memorial Hospital, Bangkok, Thailand between May 2010 and December 2015. The diagnosis of HBV infection was confirmed by the presence of serum hepatitis B $s$ antigen (HBsAg). HCC was diagnosed on the basis of typical imaging studies and/or histopathology (fine needle aspiration, core liver biopsy or surgical resection) according to the standard guideline. ${ }^{19}$ Diagnostic criteria of HCC by imaging studies were based on findings of focal hepatic lesions with hyperattenuation at the arterial phase, hypoattenuation at the portal phase in dynamic CT or MRI. The clinical parameters of patients with HCC at initial diagnosis were collected, which included sex, age, liver function tests, Child-Pugh classification, serum alpha-fetoprotein (AFP) level and HCC staging classified by the Barcelona Clinic Liver Cancer (BCLC) system. $^{20}$

Patients with chronic HBV infection, who had no evidence of HCC were recruited as the non-HCC group. These patients attended King Chulalongkorn Memorial Hospital and had been followed up every 4-6 months during the same period as patients with HCC. Chronic HBV infection was diagnosed by the positivity of serum HBsAg at least 6 months. Exclusions criteria for this group were as follows: (1) co-infection with HCV and/or human immunodeficiency virus (HIV); (2) evidence of other malignancies or autoimmune disorders during follow-up. Patients with chronic HBV infection were classified into inactive carrier (IC) and immune active (IA) phased based on the criteria of the American Association for the Study of Liver Diseases (AASLD). ${ }^{21}$ Moreover, healthy individuals recruited from blood donors at National Blood Centre Thai Red Cross Society, Bangkok, Thailand were used as the healthy control group.

The study was approved by the Institutional Review Board of the Faculty of Medicine, Chulalongkorn University, Bangkok, Thailand (IRB no. 438/60) and participants had provided written informed consent. The study followed the Helsinki Declaration and Good Clinical Practice guidelines.

\section{Plasma BAFF levels and HBV marker assays}

Baseline plasma BAFF levels were determined by ELISA (R\&D Systems) according to the manufacturer's protocol. Qualitative measurements of $\mathrm{HBsAg}$ and $\mathrm{HBeAg}$ were tested by commercial available enzyme-linked immunosorbent assays (Abbott Laboratories, Chicago, IL). HBV DNA levels were tested by Abbott Real Time HBV assay (Abbott Laboratories).

\section{Genotyping of BAFF polymorphism}

Genomic DNA was extracted from $100 \mu \mathrm{l}$ of peripheral blood mononuclear cells (PBMCs) by the phenol-chloroform isolation method according to the standard method. The quality of DNA was then measured using spectrophotometer (NanoDrop 2000c, Thermo Scientific). Genotyping of rs9514828 was performed by using polymerase chain reaction (PCR) with restriction fragment length polymorphism analysis. PCR was performed by using PCR master mix (Thermo scientific) and primers were 5'-GGCACAGTCAACATGGGAGT-3' (forward) and 5'-GCTAAGTGTTTTAGCATTGAATTG-3' (reverse) according to the previous study. ${ }^{16}$ A thermal condition was initial denaturation at $95^{\circ} \mathrm{C}$ for 3 min, followed by 40 cycles of $95^{\circ} \mathrm{C} 30 \mathrm{sec}, 58^{\circ} \mathrm{C}$ for $30 \mathrm{sec}$ and $72^{\circ} \mathrm{C}$ for $1 \mathrm{~min}$ and a final extension at $72^{\circ} \mathrm{C}$ for $7 \mathrm{~min}$. The PCR product was digested by BSrBI (New England Biolabs) and followed by $2 \%$ agarose gel electrophoresis. Another BAFF genotyping rs12583006 was performed with real-time PCR based on TaqMan genotyping assay (C_11705495_10, Applied Biosystem). The reaction was performed by using TaqMan genotyping master mix (Applied Biosystems) and 20X primers and probes mixture (TaqMan SNP Genotyping Assay, Applied Biosystems). The real-time PCR condition was performed in ABI 7500 Real Time PCR System 
(Applied Biosystems) according to the manufacturer's protocol. A thermal condition was as follow, $10 \mathrm{~min}$ at $95^{\circ} \mathrm{C}$ was hold for an initial denaturation, then followed by 40 cycles of amplification including denaturation at $95^{\circ} \mathrm{C}$ for $15 \mathrm{sec}$, and annealing/extension at $60^{\circ} \mathrm{C}$ for $1 \mathrm{~min}$. Positive and negative controls were included in each experiment in order to confirm the results.

\section{Statistical analysis}

Statistical analysis was performed with SPSS statistics version 22 (SPSS Inc., Chicago, IL) and GraphPad Prism v5.0 (GraphPad Software, San Diego, CA). Values are presented as mean \pm standard deviation (SD), and percentages as appropriate. Comparisons between groups were assessed by the $\chi 2$, Student's t-test or Bonferroni correction method for quantitative variables. Correlations between parameters were analyzed by the Pearson correlation. Survival curves in patients with HCC were established using the Kaplan-Meier method and differences between curves were assessed by the log-rank test. The Cox regression analysis was performed to identify independent factors associated with overall survival (OS) of patients with HCC. $\mathrm{P}$ values $<0.05$ were indicated statistical significance.

\section{Results \\ Clinical characteristics}

Table 1 compares baseline characteristics of all subjects enrolled in this study. Patients with HCC were older and had male gender distribution than patients without HCC and healthy controls $(P<0.001)$. Compared with the non-HCC group, patients with HCC had higher mean aspartate aminotransferase (AST), total bilirubin (TB), serum albumin, platelet counts, and AFP levels. In addition, patients with HCC had higher fibrosis-4 (FIB-4) index, a non-invasive scoring system for assessing liver fibrosis, and a higher frequency of cirrhosis than the non-HCC group. However, there was no difference between groups in terms of alanine aminotransferase (ALT), HBV DNA level and HBeAg positivity.

\section{Comparison of plasma BAFF levels between studied groups}

Plasma BAFF levels in patients with HCC obtained at the time of diagnosis ranged from 288.8 to $79.8 \mathrm{pg} / \mathrm{ml}$, with a mean of $1330.7 \pm 793.2 \mathrm{pg} / \mathrm{ml}$. The average level of plasma BAFF levels in this group was significantly higher than that of the non-HCC group $(906.5 \pm 275.6 \mathrm{pg} / \mathrm{ml}$; ranged from 476.0 to $3410.0 \mathrm{pg} / \mathrm{ml})$ and healthy controls $(845.7 \pm 158.1$ $\mathrm{pg} / \mathrm{ml}$; ranged from 487.5 to $1165.7 \mathrm{pg} / \mathrm{ml}, P<0.001)$. Plasma BAFF level in the non-HCC group was also higher than in the healthy controls $(P=0.037)$ (Figure 1A).

\section{Plasma BAFF levels in subgroups of patients without HCC}

Among the non-HCC group, patients whose clinical feature categorized in the IA phase $(\mathrm{n}=190)$ had significantly higher mean BAFF level than those classified in the IC phase $(\mathrm{n}=100)(930.5 \pm 315.3 \mathrm{pg} / \mathrm{ml}$ vs. $860.9 \pm 169.4 \mathrm{pg} / \mathrm{ml}, P=$ 0.015). If categorized patients based on HBeAg status, patients with $\mathrm{HBeAg}$ positivity $(\mathrm{n}=95)$ had significantly higher mean BAFF level than those with HBeAg negativity $(\mathrm{n}=195)(991.0$ $\pm 401.4 \mathrm{pg} / \mathrm{ml}$ vs. $865.4 \pm 172.9 \mathrm{pg} / \mathrm{ml}, P=0.004)$. Likewise, patients with cirrhosis $(n=52)$ exhibited higher average BAFF level than those without cirrhosis $(\mathrm{n}=238)(1047.6 \pm$ $440.0 \mathrm{pg} / \mathrm{ml}$ vs. $875.7 \pm 213.6 \mathrm{pg} / \mathrm{ml}, P=0.008$ ) (Figure 1B).

In the non-HCC group, plasma BAFF levels were positively correlated with AST $(\mathrm{r}=0.371, P<0.001)$, ALT $(\mathrm{r}=$ $0.435, P<0.001)$, HBV DNA $(\mathrm{r}=0.140, P=0.021)$, AFP $(\mathrm{r}$ $=0.481, P<0.001)$ and FIB-4 index $(r=0.362, P<0.001)$.

Table 1. Baseline characteristics of all subjects in the study

\begin{tabular}{|c|c|c|c|c|}
\hline Baseline Characteristics & $\begin{array}{l}\text { Healthy controls } \\
\qquad(\mathrm{n}=100)\end{array}$ & $\begin{array}{l}\text { Patients without HCC } \\
\qquad(\mathrm{n}=290)\end{array}$ & $\begin{array}{l}\text { Patients with HCC } \\
\qquad(\mathrm{n}=200)\end{array}$ & $\boldsymbol{P}$ \\
\hline Age (years) & $49.3 \pm 5.2$ & $42.9 \pm 11.8$ & $58.1 \pm 11.9$ & $<0.001^{\star}$ \\
\hline Gender (Male) & $65(65.0)$ & $174(60.0)$ & $168(84.0)$ & $<0.001^{\star}$ \\
\hline Aspartate aminotransferase (IU/L) & & $39.6 \pm 35.9$ & $95.6 \pm 102.2$ & $<0.001^{*}$ \\
\hline Alanine aminotransferase (IU/L) & & $58.9 \pm 70.3$ & $59.5 \pm 54.3$ & 0.915 \\
\hline Serum albumin (g/dL) & & $4.4 \pm 0.4$ & $3.6 \pm 0.6$ & $<0.001^{\star}$ \\
\hline Total bilirubin (mg/dL) & & $0.7 \pm 0.3$ & $1.2 \pm 0.7$ & $<0.001^{\star}$ \\
\hline Platelet count $\left(10^{9} / \mathrm{L}\right)$ & & $228.6 \pm 54.4$ & $200.0 \pm 126.9$ & $0.003^{*}$ \\
\hline HBeAg positivity & & $95(33.0)$ & $58(29.0)$ & 0.468 \\
\hline $\log _{10} \mathrm{HBV}$ DNA (IU/mL) & & $4.8 \pm 2.2$ & $4.5 \pm 1.5$ & 0.199 \\
\hline Alpha fetoprotein (ng/mL) & & $5.3 \pm 14.5$ & $17203.5 \pm 60745.5$ & $0.007^{\star}$ \\
\hline FIB-4 index & & $1.26 \pm 0.83$ & $4.87 \pm 4.14$ & $<0.001^{\star}$ \\
\hline Presence of cirrhosis & & $52(17.9)$ & $168(84.0)$ & $<0.001^{\star}$ \\
\hline BCLC stage (0-A/B/C-D) & & - & $61(30.5) / 76(38.0) / 3(31.5)$ & - \\
\hline
\end{tabular}

Data expressed as mean $\pm \mathrm{SD}$ or $\mathrm{n}(\%)$ as appropriate; ${ }^{*}, P$-value $<0.05$ 

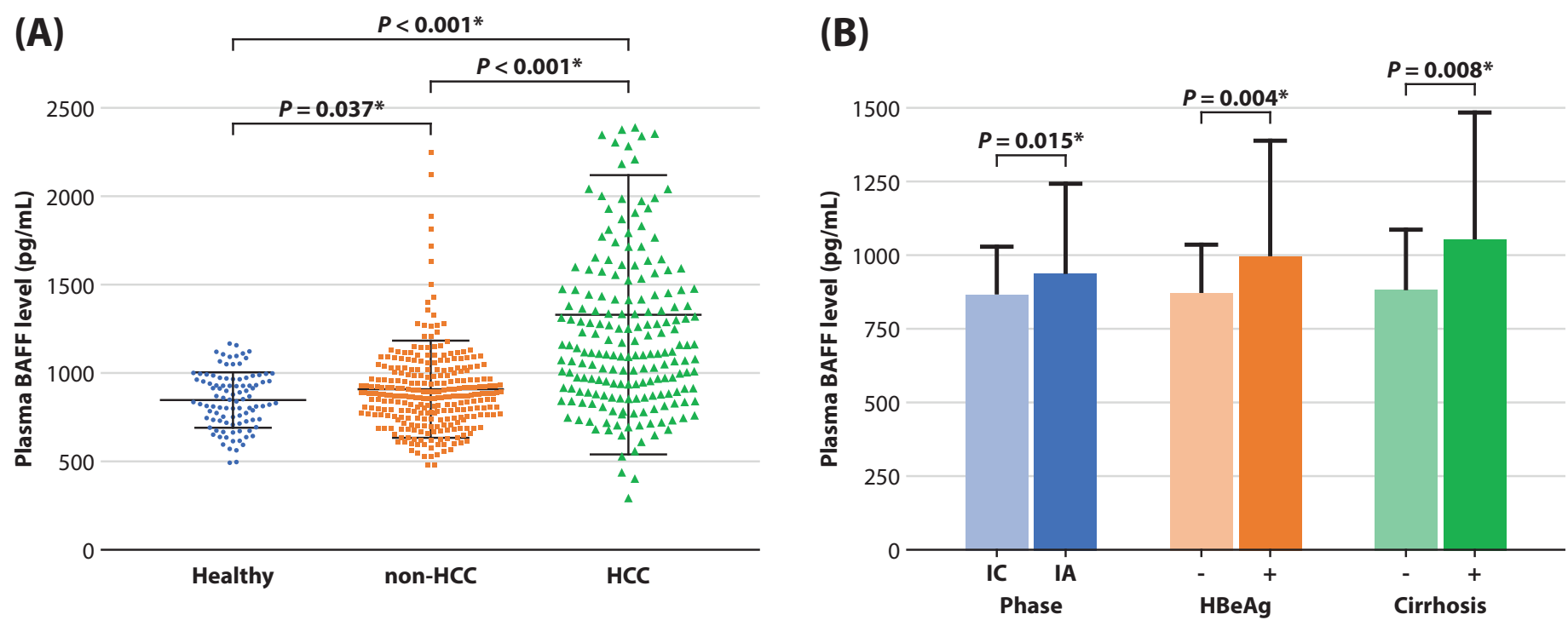

Figure 1. Plasma BAFF levels (A) Each group of patients and healthy controls (B) Subgroups of patients without HCC

Table 2. Relationship between plasma BAFF levels and characteristics of patients with HCC

\begin{tabular}{|c|c|c|c|}
\hline Variables & $\begin{array}{c}\text { Low BAFF }(<1100 \mathrm{pg} / \mathrm{ml}) \\
(\mathbf{n}=99)\end{array}$ & $\begin{array}{l}\text { High BAFF }(\geq 1100 \mathrm{pg} / \mathrm{ml}) \\
\qquad(\mathrm{n}=101)\end{array}$ & $\boldsymbol{P}$ \\
\hline Age (years) & $58.2 \pm 11.9$ & $58.0 \pm 11.9$ & 0.900 \\
\hline Gender & & & 0.177 \\
\hline Male $(\mathrm{n}=168)$ & $87(87.9)$ & $81(80.2)$ & \\
\hline Female $(\mathrm{n}=32)$ & $12(12.1)$ & $20(19.8)$ & \\
\hline Aspartate aminotransferase (IU/L) & $72.4 \pm 71.8$ & $118.4 \pm 121.1$ & $0.001^{*}$ \\
\hline Alanine aminotransferase (IU/L) & $59.3 \pm 17.5$ & $59.8 \pm 51.2$ & 0.954 \\
\hline Serum albumin $(\mathrm{g} / \mathrm{dL})$ & $3.8 \pm 0.6$ & $3.4 \pm 0.5$ & $<0.001^{\star}$ \\
\hline Total bilirubin (mg/dL) & $1.0 \pm 0.6$ & $1.3 \pm 0.8$ & $0.008^{\star}$ \\
\hline Platelet count $\left(10^{9} / \mathrm{L}\right)$ & $188.8 \pm 121.7$ & $210.9 \pm 131.5$ & 0.221 \\
\hline $\log _{10} \mathrm{HBV}$ DNA (IU/mL) & $4.5 \pm 1.5$ & $4.4 \pm 1.5$ & 0.879 \\
\hline Alpha fetoprotein (ng/mL) & $5210.3 \pm 16801.2$ & $28735.4 \pm 8208.0$ & $0.016^{*}$ \\
\hline FIB-4 index & $4.26 \pm 3.92$ & $5.46 \pm 4.28$ & 0.069 \\
\hline \multicolumn{4}{|l|}{ Child-Puge class } \\
\hline$A(n=158)$ & $89(87.3)$ & $69(70.4)$ & $0.027^{\star}$ \\
\hline B or C $(n=42)$ & $13(12.7)$ & $29(29.6)$ & \\
\hline \multicolumn{4}{|l|}{ BCLC tumor stage } \\
\hline $0-A(n=61)$ & $40(40.4)$ & $21(20.8)$ & $<0.001^{*}$ \\
\hline$B(n=76)$ & $41(41.4)$ & $35(34.7)$ & \\
\hline C-D $(n=63)$ & $18(18.2)$ & $45(44.6)$ & \\
\hline
\end{tabular}

Data expressed as mean $\pm \mathrm{SD}$ or $\mathrm{n}(\%)$ as appropriate; ${ }^{\star}, P$-value $<0.05$ 


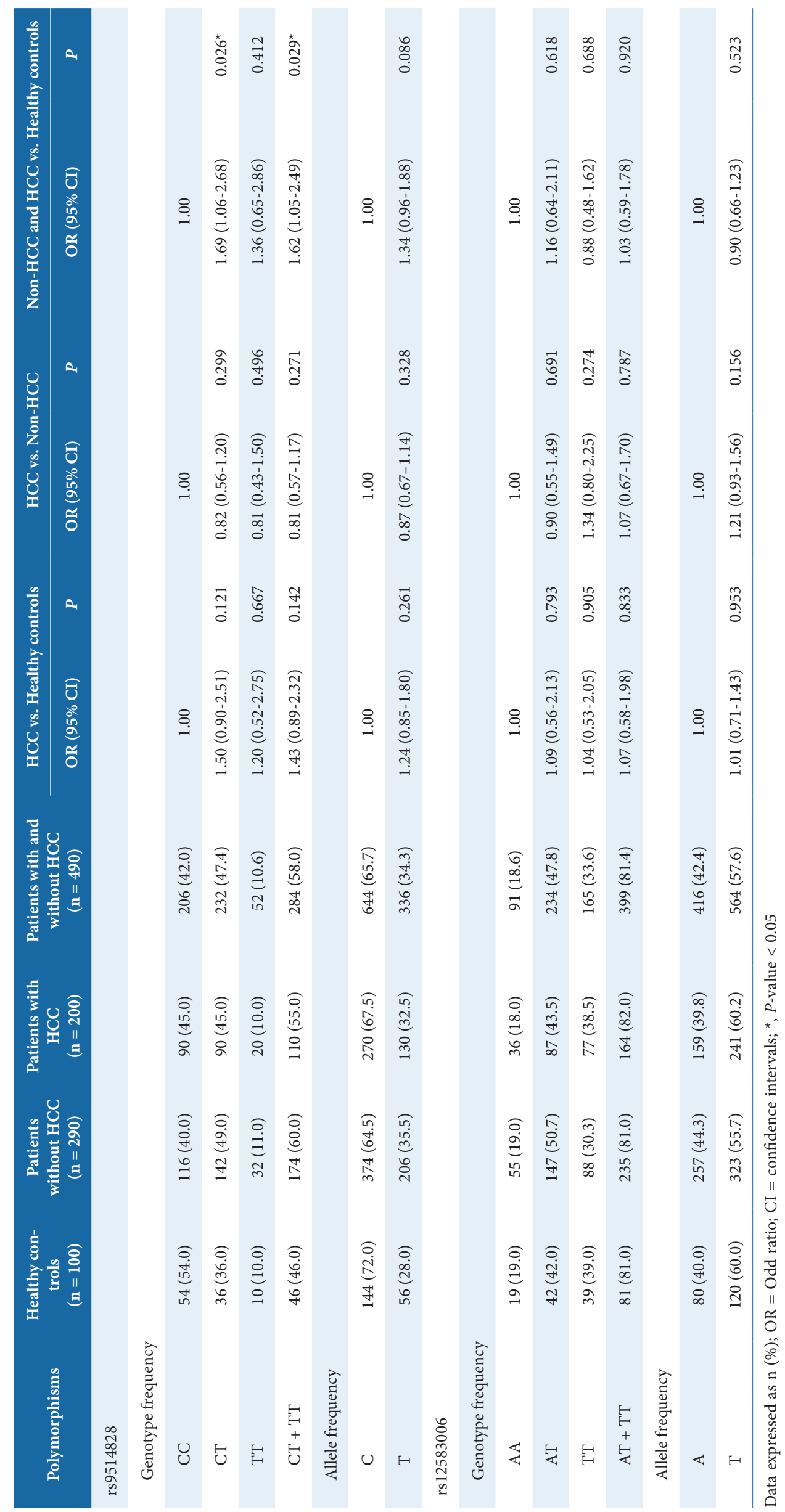


There was no correlation between plasma BAFF levels and other clinical parameters (age, sex, total bilirubin, platelet counts and serum albumin).

\section{Plasma BAFF levels and clinical features in patients with HCC}

To evaluate the association between plasma BAFF levels and clinical features, the patients with HCC were divided into two groups based on their median value (approximately $1100 \mathrm{pg} / \mathrm{ml}$ ) in all HCC patients. Accordingly, there were 99 and 101 patients with low and high levels of plasma BAFF, respectively. The correlations of low and high BAFF levels and various clinical parameters are summarized in Table 2. It was clearly shown that high BAFF levels were significantly correlated with serum AFP, severity of liver disease determined by Child-Pugh Classification and advanced BCLC stage. However, there was no correlation between plasma BAFF level and patient age, gender, platelet count, HBV DNA level and FIB-4 index.

\section{Distribution of BAFF polymorphisms}

Prevalence of the SNPs in the BAFF gene including rs9514828 and rs12583006 in each group of subjects are summarized in Table 3. There was no difference in the prevalence of rs9514828 genotypes between patients with HCC and nonHCC, as well as between patients with HCC and healthy controls. However, patients with chronic HBV infection (including HCC and non-HCC) had a significantly higher prevalence of CT and CT + TT compared with healthy controls. Regarding rs12583006 genotypes, there was no difference in their distribution among studied groups.

The associations between these two SNPs and plasma BAFF levels were also examined. However, our data did not detect any significant difference of plasma BAFF levels in relation to different genotypes of rs9514828 or rs12583006 in all subjects. For example, average BAFF level of individuals with rs9514828 CT and CT + TT was $1054.5 \pm 630.2$ and $1028.6 \pm 469.6 \mathrm{pg} / \mathrm{ml}$, respectively $(P=0.580)$, while average BAFF level of individuals with rs12583006 AA and AT + TT was $1113.2 \pm 802.7$ and $1023.2 \pm 467.1 \mathrm{pg} / \mathrm{ml}$, respectively $(P$ $=0.259$ ). Also, there was no such difference in subgroups of patients with HCC, patients without HCC and healthy controls (data not shown).

Factors associated with overall survival of patients with HCC

We further examined the potential prognostic value of plasma BAFF and its related SNPs. The median overall survival (OS) of patients with low levels of BAFF $(<1100 \mathrm{pg} / \mathrm{ml})$ was 47.5 months, which was significantly better than that of patients whose levels were $\geq 1100 \mathrm{pg} / \mathrm{ml}$ (21.4 months, $P<$ 0.001 by $\log$ rank test) (Figure 2A). For rs9514828, there was no difference in OS between patients harboring CC or CT + TT (Figure 2B). Similarly, there was no difference in OS between patients harboring AA or AT + TT of rs12583006 (Figure $2 \mathrm{C})$.

Plasma BAFF, rs9514828 and rs12583006 were entered into multivariate analysis together with other variables that might influence OS of patients with HCC. These factors included age, gender, AST, ALT, platelet count, HBV DNA, FIB-4 index, Child-Pugh classification, tumor size and BCLC stage. The multivariate analysis revealed that more advanced BCLC (stage B,C,D vs stage 0,A) [odds ratio $(\mathrm{OR})=3.00$, 95\% confidence intervals (CI):1.53-5.87; $P=0.001]$, high $\operatorname{AFP}(\geq 100$ vs. $<100 \mathrm{ng} / \mathrm{ml})(\mathrm{OR}=3.64,95 \% \mathrm{CI}: 1.53-8.64$; $P=0.003)$ and high plasma BAFF levels $(\geq 1100$ vs. $<1100$ $\mathrm{pg} / \mathrm{ml})(\mathrm{OR}=2.28,95 \% \mathrm{CI}: 1.07-4.87 ; \mathrm{P}=0.034)$ were independent poor prognostic factors of OS in patients with HCC (Table 4).
(A)

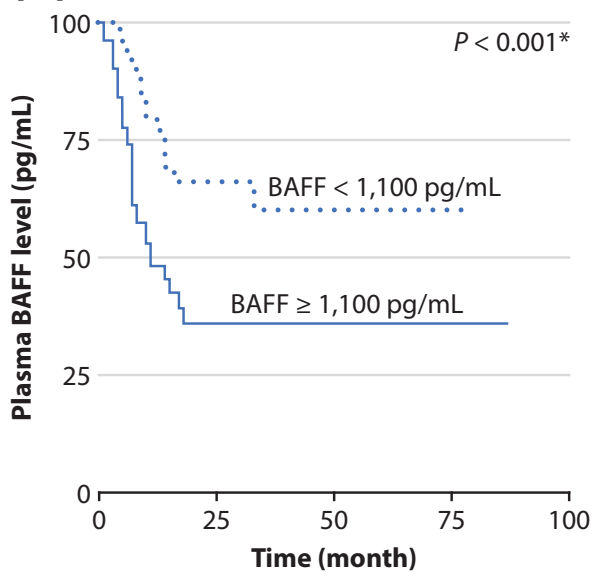

(B)

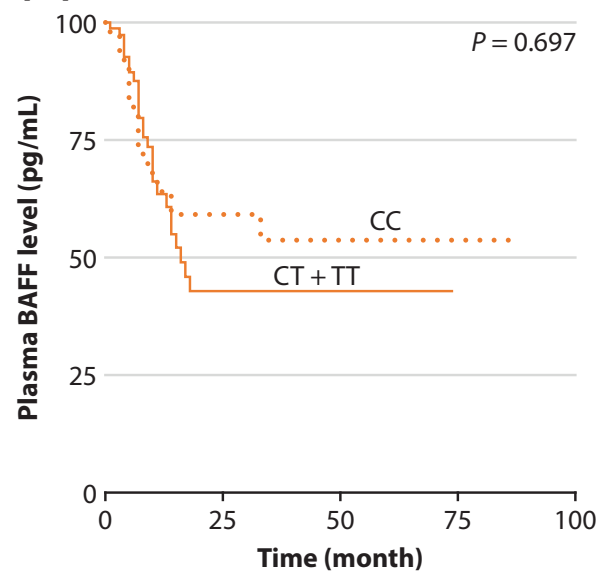

(C)

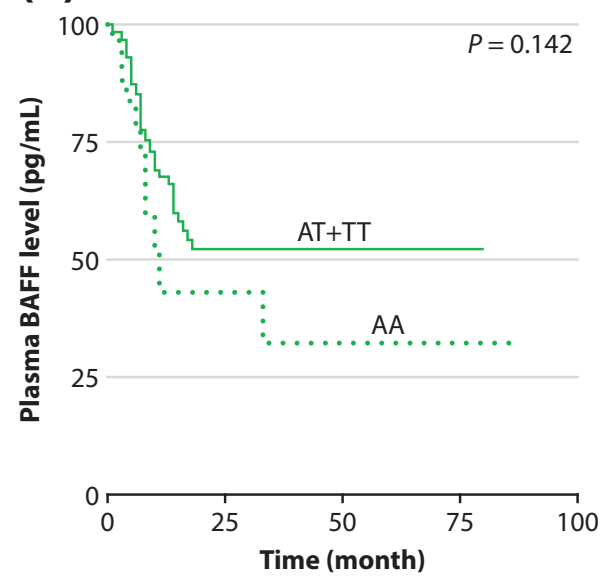

Figure 2. Overall survivals of patients with HCC regarding to BAFF levels and polymorphisms (A) plasma BAFF levels (B) rs9514828 genotypes (C) rs12583006 genotypes 
Table 4. Factors associated with overall survival in patients with HCC

\begin{tabular}{|c|c|c|c|c|c|}
\hline \multirow{3}{*}{ Factors } & \multirow{3}{*}{ Category } & \multicolumn{4}{|c|}{ Overall survival } \\
\hline & & \multicolumn{2}{|c|}{ Univariate analysis } & \multicolumn{2}{|c|}{ Multivariate analysis } \\
\hline & & OR $(95 \% \mathrm{CI})$ & $P$ & OR $(95 \% \mathrm{CI})$ & $\boldsymbol{P}$ \\
\hline Age (years) & $<60$ vs. $\geq 60$ & $1.98(1.14-3.45)$ & $0.016^{*}$ & $0.69(0.30-1.60)$ & 0.387 \\
\hline Gender & Male vs. Female & $1.35(0.70-2.61)$ & 0.374 & & \\
\hline Aspartate aminotransferase (IU/L) & $<60$ vs. $\geq 60$ & $3.14(1.76-5.63)$ & $<0.001^{*}$ & $0.92(0.39-2.19)$ & 0.853 \\
\hline Alanine aminotransferase (IU/L) & $<60$ vs. $\geq 60$ & $2.74(1.62-4.65)$ & $<0.001^{*}$ & $1.34(0.63-2.82)$ & 0.445 \\
\hline Platelet count $\left(10^{9} / \mathrm{L}\right)$ & $\geq 150$ vs. $<150$ & $2.94(1.55-5.57)$ & $0.001^{*}$ & $1.94(0.75-5.02)$ & 0.174 \\
\hline $\log _{10} \mathrm{HBV}$ DNA $(\mathrm{IU} / \mathrm{mL})$ & $<4.0$ vs. $\geq 4.0$ & $0.78(0.39-1.56)$ & 0.475 & & \\
\hline Child-Pugh classification & A vs. B and C & $1.42(0.67-3.02)$ & 0.361 & & \\
\hline Alpha fetoprotein (ng/mL) & $<100$ vs. $\geq 100$ & $5.91(2.99-11.68)$ & $<0.001^{*}$ & $3.64(1.53-8.64)$ & $0.003^{*}$ \\
\hline FIB-4 index & $<3.40$ vs. $\geq 3.40$ & $0.87(0.52-1.51)$ & 0.656 & & \\
\hline Tumor size (cm.) & $<5.0$ vs. $\geq 5.0$ & $10.55(4.69-23.75)$ & $<0.001^{*}$ & $2.10(0.62-7.10)$ & 0.231 \\
\hline BCLC stage & $0, A$ vs. $B, C, D$ & $4.42(2.91-6.70)$ & $<0.001^{*}$ & $3.00(1.53-5.87)$ & $0.001^{*}$ \\
\hline Plasma BAFF level (pg/ml) & $<1100$ vs. $\geq 1100$ & $3.10(1.75-5.49)$ & $<0.001^{*}$ & $2.28(1.07-4.87)$ & $0.034^{*}$ \\
\hline rs9514828 & $\mathrm{CC}$ vs. $\mathrm{CT}+\mathrm{TT}$ & $1.32(0.52-3.32)$ & 0.557 & & \\
\hline rs12583006 & AA vs. AT + TT & $0.98(0.57-1.67)$ & 0.931 & & \\
\hline
\end{tabular}

Data express as odds ratio (OR) and $95 \%$ confidence intervals $(\mathrm{CI}) ;{ }^{*}, P$-value $<0.05$

\section{Discussion}

HCC represents a leading cancers worldwide, especially in Southeast Asia, where HBV is highly prevalent. ${ }^{1}$ Overall, the prognosis of HCC is poor due to aggressive tumor characteristics and an advanced stage at presentation. The pathogenesis of HBV-related HCC is thought to be a multi-step process, which is mainly associated with immune-mediated liver injury. $^{2}$ Accumulating evidence has demonstrated the critical roles of adaptive immunity response to HBV infection that contributes in chronic liver inflammation leading to hepatocarcinogenesis, though most reports have focused on the effects of HBV-specific T cells. ${ }^{22}$ Conversely, relatively little is currently known regarding the clinical importance of B-cell immunity in HCC progression and prognosis.

In this study, we confirmed previous data that circulating BAFF levels were significantly higher in patients with HCC compared with healthy individuals and patients with nonHCC. ${ }^{14}$ Among patients without HCC, plasma BAFF levels had a gradually increase from inactive carriers (IC) to the immune active (IA) group and cirrhosis. Our data showed that plasma BAFF levels were positively correlated with serum ALT, a surrogate marker of liver injury. These data indicate that BAFF is increased during active inflammation, possibly as a consequence of BAFF production by its inducers such as type I interferons. ${ }^{5}$ Notably, among patients with immune active disease, BAFF levels were significantly higher in patients with $\mathrm{HBeAg}$-positive that those with $\mathrm{HBeAg}$-negative. Consistent with our report, previous data from a cell culture model demonstrated that $\mathrm{HBeAg}$ itself was capable of promoting BAFF activation through regulating monocyte function. ${ }^{23}$
Our results also demonstrated that BAFF levels correlated with liver fibrosis assessed by FIB-4 index and their levels were significantly increased in patients with cirrhosis compared with the non-cirrhotic group, supporting previous findings that BAFF levels progressively increase in cirrhosis independent of underlying etiologies of liver disease..$^{22,24}$

Our data showed that elevated plasma BAFF levels were significantly correlated with more aggressive tumor characteristics in patients with HBV-related HCC. In addition, a high BAFF level at initial presentation was associated with an unfavorable outcome in these patients. Specifically, a high plasma BAFF level was observed more frequently in patients with large tumor burden and advanced BCLC stages. Furthermore, multivariate analysis revealed that this marker was an independent, unfavorable predictor of survival in patients with HCC. Specifically, HCC patients with high circulating BAFF $(\geq 1100 \mathrm{pg} / \mathrm{ml})$ at initial presentation had approximately 2 -fold increased risk of adverse outcome compared to patients with lower BAFF levels. These data strongly suggest that the prognosis of HCC is influenced by the extent of circulating BAFF expression. Collectively, these findings demonstrate that plasma BAFF may represent a useful biomarker in monitoring tumor progression and prognosis in patients with HBV-related HCC.

Similar findings of BAFF levels in association with tumor progression and prognosis were observed in hematological and non-hematological malignancies. ${ }^{10,25-28}$ For instance, the levels of BAFF were positively correlated with disease severity, poor therapeutic response and adverse clinical outcome 
in patients with lymphoma. ${ }^{10,25,26}$ In addition, BAFF levels were associated with disease activity and advanced disease stage in patients with multiple myeloma. ${ }^{27}$ Moreover, circulating BAFF levels were highly expressed in patients with pancreatic cancer, especially among those with metastatic disease. ${ }^{27}$ Significantly elevated circulating BAFF were also found in adolescent patients with certain types of sarcoma in relation to cancer-related cachexia. ${ }^{28}$ These data highlight an essential and active role of BAFF in disease severity of HCC and other tumor types.

As BAFF has emerged a critical factor of peripheral B cell survival, it is likely that B cells may be contributable to disease progression and HCC development in patients with chronic HBV infection. To support this notion, a previous study directly demonstrated that $\mathrm{B}$ celldeficient mice displayed attenuated liver fibrosis induced by $\mathrm{CCl} 4$, representing a pro-fibrogenic activity of $\mathrm{B}$ cells. ${ }^{29} \mathrm{~A}$ more recent report showed that intrahepatic B cells were responsible for hepatic stellate cell-mediated liver fibrosis through the production of several inflammatory cytokines. ${ }^{30}$ Moreover, a recent study showed that B cells played a critical role in hepatocarcinogenesis following chronic liver injury. ${ }^{31}$ In an animal model, elimination of $\mathrm{B}$ cells, but not $\mathrm{T}$ cells, could promote the resolution of liver fibrosis and prevent important signaling pathways towards HCC development. The role of B cells was also confirmed in patients with HCC demonstrating that increased infiltrating B cells within cancerous tissues was linked to poor tumor differentiation, advanced stages and reduced disease-free survival of HCC. ${ }^{31}$ Likewise, increase percentage of B cells in PBMCs was also demonstrated in patients with more advanced tumor stages compared to those with early HCC. ${ }^{26}$ Taken together, these data highlight the significance of $\mathrm{B}$ cells in modulating liver fibrogenesis and, more importantly, the development and progression of HCC.

Regarding the BAFF polymorphisms, it was previously shown that these genetic variations might result in changes of BAFF activity and expression, and was reported to be associated with the pathogenesis of autoimmune diseases, hematological malignancies or chronic infection. ${ }^{15-17}$ For instance, the $\mathrm{T}$ allele of rs9514828 polymorphism in the BAFF promoter was more predominant in patients with HCV-related mixed cryoglobulinemia (MC) and associated with an increase in BAFF levels when compared with chronic $\mathrm{HCV}$ carriers without MC. ${ }^{32,33}$ In this study, our data showed that the frequency of rs9514828 CT + TT genotypes was significantly higher distributed in patients with chronic HBV infection, including the HCC and non-HCC groups, compared with healthy controls. However, significant difference in their distributions between patients with HCC and non-HCC was not observed. Moreover, rs9514828 genotypes exhibited no association with plasma BAFF levels or other clinical parameters of patients with chronic HBV infection. These results suggested that $\mathrm{CT}+\mathrm{TT}$ genotypes might be associated with susceptibility to $\mathrm{HBV}$ infection but not related to disease progression or HCC development in Thai populations. Of noted, our findings were partly in line with recent data demonstrating that these genotypes might confer susceptibility to chronic HBV infection in Chinese Han populations, probably not directly through circulating BAFF expression. ${ }^{18}$
Regarding rs12583006, another polymorphism located in the noncoding region of BAFF, our results showed that this genetic variation did not have any influence on plasma BAFF levels and, more importantly, displayed no role on clinical significance in patients with chronic HBV infection.

This report might have some limitations. First, the study was a retrospective design and the sample size of patients with or without HCC was relatively small. Second, the analysis of genetic variations included only two polymorphisms and limited in Thai patients, which might not be applicable to other ethnic populations.

\section{Conclusion}

In conclusion, this is the first report demonstrates the clinical implications of plasma BAFF in patients with HBV-related HCC. We found that a high level of BAFF was significantly associated with tumor progression and invasiveness. Moreover, elevated plasma BAFF level was an independent prognostic factor of overall survival. These findings have clinical implications as plasma BAFF at initial diagnosis could serve as a prognostic marker for patients with HBV-related HCC. Also, these data might indicate that B cell immunity is contributable to the development and progression of HCC in patients with chronic HBV infection. Further studies are, however, required to validate these observations in patients with HCC regardless of underlying etiologies and to elucidate the mechanistic roles of $\mathrm{B}$ cell-mediated immune response in hepatocarcinogenesis.

\section{Acknowledgements}

This study was funded by the Grant for Chula Research Scholar [CU-GRS-60-06-30-03], the 100 ${ }^{\text {th }}$ Anniversary Chulalongkorn University Fund for Doctoral Scholarship, the $90^{\text {th }}$ Anniversary Chulalongkorn University Fund (Ratchadaphiseksomphot Endowment Fund), Chulalongkorn University, the annual government budget in the year 2017-2018 and The Thailand Research Fund (TRF) Senior Research Scholar (RTA5980008). The work was also supported by the Center of Excellence in Hepatitis and Liver Cancer, Faculty of Medicine, Chulalongkorn University.

\section{Conflict of Interest}

All authors declare no conflict of interest.

\section{References}

1. Trepo C, Chan HL, Lok A. Hepatitis B virus infection. Lancet. 2014; 384:2053-63.

2. Bertoletti A, Ferrari C. Innate and adaptive immune responses in chronic hepatitis B virus infections: towards restoration of immune control of viral infection. Gut. 2012;61:1754-64.

3. Akram N, Imran M, Noreen M, Ahmed F, Atif M, Fatima Z, et al. Oncogenic Role of Tumor Viruses in Humans. Viral Immunol. 2017;30: 20-7.

4. Bertoletti A, Ferrari C. Adaptive immunity in HBV infection. J Hepatol. 2016;64:S71-83.

5. Lu L. Frontiers in B-cell immunology. Cell Mol Immunol. 2013;10:95-6.

6. Mackay F, Browning JL. BAFF: a fundamental survival factor for B cells. Nat Rev Immunol. 2002;2:465-75.

7. Lied GA, Berstad A. Functional and clinical aspects of the B-cell-activating factor (BAFF): a narrative review. Scand J Immunol. 2011;73:1-7. 
8. Cheema GS, Roschke V, Hilbert DM, Stohl W. Elevated serum B lymphocyte stimulator levels in patients with systemic immune-based rheumatic diseases. Arthritis Rheum. 2001;44:1313-9.

9. Salazar-Camarena DC, Ortiz-Lazareno PC, Cruz A, Oregon-Romero E, Machado-Contreras JR, Munoz-Valle JF, et al. Association of BAFF, APRIL serum levels, BAFF-R, TACI and BCMA expression on peripheral B-cell subsets with clinical manifestations in systemic lupus erythematosus. Lupus. 2016;25:582-92.

10. Novak AJ, Grote DM, Stenson M, Ziesmer SC, Witzig TE, Habermann TM, et al. Expression of BLyS and its receptors in B-cell non-Hodgkin lymphoma: correlation with disease activity and patient outcome. Blood. 2004;104:2247-53.

11. Sakai J, Akkoyunlu M. The Role of BAFF System Molecules in Host Response to Pathogens. Clin Microbiol Rev. 2017;30:991-1014.

12. Lake-Bakaar G, Jacobson I, Talal A. B cell activating factor (BAFF) in the natural history of chronic hepatitis $\mathrm{C}$ virus liver disease and mixed cryoglobulinaemia. Clin Exp Immunol. 2012;170:231-7.

13. Sene D, Limal N, Ghillani-Dalbin P, Saadoun D, Piette JC, Cacoub P. Hepatitis C virus-associated B-cell proliferation--the role of serum B lymphocyte stimulator (BLyS/BAFF). Rheumatology (Oxford). 2007;46: 65-9.

14. Yang C, Li N, Wang Y, Zhang P, Zhu Q, Li F, et al. Serum levels of B-cell activating factor in chronic hepatitis B virus infection: association with clinical diseases. J Interferon Cytokine Res. 2014;34:787-94.

15. Jasek M, Bojarska-Junak A, Wagner M, Sobczynski M, Wolowiec D, Rolinski J, et al. Association of variants in BAFF (rs9514828 and rs1041569) and BAFF-R (rs61756766) genes with the risk of chronic lymphocytic leukemia. Tumour Biol. 2016;37:13617-26.

16. Novak AJ, Slager SL, Fredericksen ZS, Wang AH, Manske MM, Ziesmer $\mathrm{S}$, et al. Genetic variation in B-cell-activating factor is associated with an increased risk of developing B-cell non-Hodgkin lymphoma. Cancer Res. 2009;69:4217-24

17. Nezos A, Papageorgiou A, Fragoulis G, Ioakeimidis D, Koutsilieris M, Tzioufas AG, et al. B-cell activating factor genetic variants in lymphomagenesis associated with primary Sjogren's syndrome. J Autoimmun. 2014;51:89-98.

18. Han Q, Yang C, Li N, Li F, Sang J, Lv Y, et al. Association of genetic variation in B-cell activating factor with chronic hepatitis B virus infection. Immunol Lett. 2017;188:53-8.

19. Bruix J, Sherman M, American Association for the Study of Liver Diseases. Management of hepatocellular carcinoma: an update. Hepatology. 2011;53:1020-2.

20. Forner A, Llovet JM, Bruix J. Hepatocellular carcinoma. Lancet. 2012;379: 1245-55.
21. Terrault NA, Bzowej NH, Chang KM, Hwang JP, Jonas MM, Murad MH, et al. AASLD guidelines for treatment of chronic hepatitis B. Hepatology. 2016;63:261-83.

22. Doi H, Hayashi E, Arai J, Tojo M, Morikawa K, Eguchi J, et al. Enhanced B-cell differentiation driven by advanced cirrhosis resulting in hyperglobulinemia. J Gastroenterol Hepatol [Preprint]. 2018. [cited 2018 Jul 16]: [10 p.]. Available from: https://doi.org/10.1111/jgh.14123

23. Lu B, Zhang B, Wang L, Ma C, Liu X, Zhao Y, et al. Hepatitis B Virus e Antigen Regulates Monocyte Function and Promotes B Lymphocyte Activation. Viral Immunol. 2017;30:35-44.

24. Miyake T, Abe M, Tokumoto Y, Hirooka M, Furukawa S, Kumagi T, et al. B cell-activating factor is associated with the histological severity of nonalcoholic fatty liver disease. Hepatol Int. 2013;7:539-47.

25. Kim SJ, Lee SJ, Choi IY, Park Y, Choi CW, Kim IS, et al. Serum BAFF predicts prognosis better than APRIL in diffuse large B-cell lymphoma patients treated with rituximab plus CHOP chemotherapy. Eur J Haematol. 2008;81:177-84.

26. Lin JC, Shih YL, Chien PJ, Liu CL, Lee JJ, Liu TP, et al. Increased percentage of $\mathrm{B}$ cells in patients with more advanced hepatocellular carcinoma. Hum Immunol. 2010;71:58-62.

27. Fragioudaki M, Boula A, Tsirakis G, Psarakis F, Spanoudakis M, Papadakis IS, et al. B cell-activating factor: its clinical significance in multiple myeloma patients. Ann Hematol. 2012;91:1413-8.

28. Bienertova-Vasku J, Lungova A, Bienert P, Zlamal F, Tomandl J, Tomandlova $\mathrm{M}$, et al. Circulating levels of B-cell activating factor in paediatric patients with malignancy with or without cancer-related cachexia. Klin Onkol. 2012;25 Suppl 2:2S58-63.

29. Novobrantseva TI, Majeau GR, Amatucci A, Kogan S, Brenner I, Casola $\mathrm{S}$, et al. Attenuated liver fibrosis in the absence of B cells. J Clin Invest. 2005; 115:3072-82.

30. Thapa M, Chinnadurai R, Velazquez VM, Tedesco D, Elrod E, Han JH, et al. Liver fibrosis occurs through dysregulation of MyD88-dependent innate B-cell activity. Hepatology. 2015;61:2067-79.

31. Faggioli F, Palagano E, Di Tommaso L, Donadon M, Marrella V, Recordati C, et al. B lymphocytes limit senescence-driven fibrosis resolution and favor hepatocarcinogenesis in mouse liver injury. Hepatology. 2018;67(5):1970-85. Epub 2018 Mar 25.

32. Gragnani L, Piluso A, Giannini C, Caini P, Fognani E, Monti $M$, et al. Genetic determinants in hepatitis $C$ virus-associated mixed cryoglobulinemia: role of polymorphic variants of BAFF promoter and Fcgamma receptors. Arthritis Rheum. 2011;63:1446-51.

33. Ayad MW, Elbanna AA, Elneily DA, Sakr AS. Association of BAFF -871C/T Promoter Polymorphism with Hepatitis C-Related Mixed Cryoglobulinemia in a Cohort of Egyptian Patients. Mol Diagn Ther. 2015;19:99-106. 